\title{
Vegetación nativa como factor de control de erosión y restauración ecológica, San José, Costa Rica
}

\section{Native vegetation as erosional control and ecological restoration, San José, Costa Rica}

\section{Virginia Alvarado-García ${ }^{1}$, María Auxiliadora Zúñiga-Amador ${ }^{2}$}

'Laboratorio de Vida y Salud, Red de Gestión en Conservación de Vida Silvestre y Salud (GeCoS), Vicerrectoría de Investigación, ORCDI: http://orcid.org/0000-0001-7430-0549 / ${ }^{2}$ Manejo de Recursos Naturales, Escuela de Ciencias Exactas y Naturales. Universidad Estatal a Distancia, Montes de Oca, Costa Rica. Autor de correspondencia: V. Alvarado [vicky1610@gmail.com]

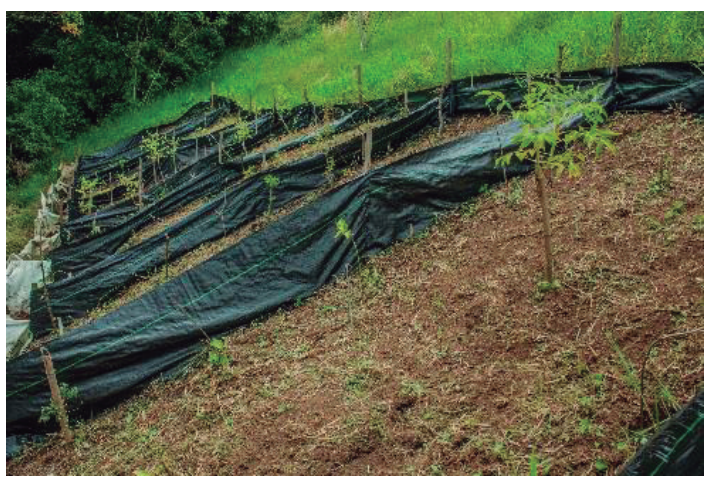

\section{RESUMEN}

El establecimiento de una capa protectora compuesta por especies nativas, que desarrollen una comunidad sucesora uniforme, es básico para la restauración ecológica y es necesario para controlar los procesos erosivos. Con el fin de evaluar especies vegetales nativas para el control de la erosión y restauración ecológica, se trabajó en tres sitios a lo largo del río Torres. Para ello, se instalaron ocho parcelas experimentales en cada sitio, una de ellas sin vegetación y el resto con diferentes especies de herbáceas y arbustos. En cada sitio se tomaron en cuenta variables edáficas y climáticas; mientras que en cada parcela, se evaluó la sobrevivencia de las especies y la producción de sedimentos de forma mensual. La sobrevivencia promedio en los tres sitios estuvo por encima del 50\%, lo cual evidencia que las especies superaron el estrés post siembra y lograron adaptarse exitosamente. En la parte alta y baja, las parcelas sembradas con Hamelia patens y Tradescantia zanonia, registraron menos tasa de erosión. Para la parte media, Dahlia imperialis y T. zanonia, fueron las más eficientes en comparación a las otras cuatro especies. En general, en la parte alta y baja, predominaron los arbustos; mientras que en la media, las herbáceas. El uso de especies nativas como estrategia de restauración ecológica y foresta urbana permite mejorar las condiciones mecánicas, hidráulicas y ambientales del sitio de estudio, y actúa como barrera viva contra la erosión.

Palabras clave: arbustos; barrera viva; foresta urbana; herbáceas; parcelas sedimentos; sobrevivencia, río Torres.
The establishment of a protective layer composed by native species to develop a uniform successor community is essential for ecological restoration and is necessary to control eroding processes. A field assessment was carried out in three sites along the Torres river watershed through the establishment of eight experimental plots per site, one of them without vegetation and the rest with different herbaceous and shrub species. Edaphic and climatic variables were considered on each site, while in each plot the species survival and the sediments production were evaluated monthly. The survival average in the three sites was over $50 \%$, showing the species overcame post-sowing stress and managed to adapt successfully. In the upper and lower part, the plots sown with Hamelia patens and Tradescantia zanonia registered a lower erosion rate. For the middle part, Dahlia imperialis and T. zanonia, were the most efficient compared to the other four species. In general, in the high and low areas, the shrubs are dominant, and the herbaceous in the middle. The use of native species as a strategy of ecological restoration and urban forestry allows the improving of mechanical, hydraulic and environmental conditions of the study site, and acts as a living barrier against erosion.

Key words: shrubs; live barrier; urban forestry; herbs; plots; sediments; survival; Torres river watershed.
Recibido: 18 de abril del 2018 Aceptado: 8 de junio del 2018

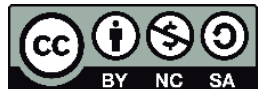

Los artículos de la revista La Calera de la Universidad Nacional Agraria, Nicaragua, se comparten bajo términos de la licencia Creative Commons: Reconocimiento, No Comercial, Compartir Igual. Las autorizaciones adicionales a las aquí delimitadas se pueden obtener en el correo freddy.aleman@ci.una.edu.ni

C Copyright 2018. Universidad Nacional Agraria 


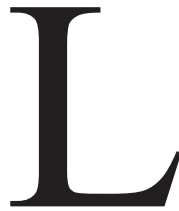

as principales variables que intervienen en los procesos erosivos son la vegetación, el clima, la topografía, el tipo y uso del suelo. De ellas, la falta de cobertura vegetal es una de las causas más influyentes en los problemas de degradación de los terrenos (Suarez, 2001).

La vegetación incrementa la protección del suelo, frena la escorrentía y facilita la infiltración. Los componentes radiculares contribuyen a aumentar la resistencia mecánica del suelo, y la presencia de materia orgánica, ofrece estabilidad, rugosidad y porosidad, lo que supone un aumento en su capacidad de infiltración (Bochet y García, 2004; Zúñiga, 2016).

El establecimiento de una capa protectora compuesta por especies nativas, que desarrollen una comunidad sucesora uniforme, es básico para la restauración de áreas degradadas (Rondón y Vidal, 2005). Es fundamental el uso de plantas autóctonas, ya que están bien adaptadas al medio, desarrollan un sistema de raíces extensivo, son excelentes para la vida silvestre propia del lugar y no requieren mucho mantenimiento (Alvarado et al., 2013).

El uso de coberturas vegetales constituye una práctica de baja inversión, pero muy efectiva como estructuras de sostén y refuerzo en terrenos inestables (Laporte y Porras, 2002); asimismo para la resistencia y conservación del suelo (Zwart et al., 2005). Desde el punto de vista práctico, ético y económico, estas alternativas son más sustentables y van en armonía con el ambiente; sin embargo, se deben considerar las mejores especies que logren adaptarse, colonizar y ser exitosas para las condiciones que se tengan.

La sobrevivencia y el crecimiento de las especies vegetales es impredecible, ambas están relacionados con las variables fisicoquímicas del suelo, la calidad del material de plantación, las técnicas de siembra, las condiciones de deterioro del sitio y la ausencia o exceso de precipitaciones que enfrentan las plantas durante el proceso de estabilización y adaptación. El estrés es determinante, puesto que hay especies capaces de soportar condiciones adversas, pero tienen tasas de crecimiento más bajas; por el contrario, especies competitivas consiguen mayor productividad en condiciones favorables (Villar, 2004).

Por lo tanto, para intervenir los sitios fragmentados por la acción antrópica, se requiere del conocimiento de las interacciones entre las condiciones urbanas, el estado del suelo, de las aguas, así como, de los instrumentos y técnicas para su manejo, mediante la implementación de técnicas de restauración ecológica y mejoramiento de ríos urbanos, que busquen recuperar las funciones ecológicas de los cursos de agua, así como la vegetación próxima al cauce mientras se mantiene el uso antropogénico de las mismas (Zúñiga, 2016).
Costa Rica no cuenta con criterios técnicos sólidos que demuestren que una especie vegetal realmente protege y refuerza el suelo de forma adecuada. La información existente en cuanto a la biología y ecología de las especies vegetales es incompleta, dispersa o inaccesible (Porras, 2000); sin embargo, ya se perfilan algunos esfuerzos por documentar estas carencias Sancho y Cervantes, 1997; Laporte y Porras, 2002; Zwart et al., 2005), y sobre todo en las ciudades (Alvarado et al., 2013).

Por otro lado, en el Gran Área Metropolitana de Costa Rica se han realizado proyectos de foresta urbana (Conejero y Sallent, 2011; Mendoza, 2012) y establecimiento de corredores fluviales verdes interurbanos en las provincias de San José (Feoli, 2013) y Heredia (Romero et al., 2011), los cuales permiten un aumento en la cobertura vegetal y la biodiversidad a través de una trama verde continua.

A pesar de ello, no se ha considerado la revegetación, como una estrategia de restauración ecológica y conectividad de la red natural urbana. Un primer acercamiento se realizó en el 2011, en donde se enlistaron más de 50 especies nativas con potencial para el control de la erosión para ríos urbanos y además, se evaluó la retención de sedimentos mediante el uso de tres especies vegetales. Se demostró que las plantas nativas son más eficientes que las exóticas y además, poseen un valor ecológico agregado (Alvarado et al., 2013).

Esta investigación pretende determinar el potencial de especies vegetales nativas para el control de la erosión en taludes de la microcuenca urbana del río Torres; así como su función en la retención de sedimentos y su potencial ecológico en ecosistemas degradados.

\section{MATERIALES Y MÉTODOS}

Zona de estudio. El estudio se realizó en la microcuenca del río Torres, San José, Costa Rica, el cual nace en Rancho Redondo de Goicochea y desemboca en la subcuenca del río Virilla, sector de La Carpio, en la Uruca. Se ubica entre los 900 y $2400 \mathrm{msnm}$ con una precipitación de 200 a $3000 \mathrm{~mm}$ anuales, temperaturas promedio es de $20^{\circ} \mathrm{C}$ y la humedad relativa media anual es de $70 \%$. Involucra Bosque muy Húmedo Montano Bajo (bmh-MB), el Bosque muy Húmedo Premontano (bmh-P) y el Bosque Húmedo Premontano (bh-P) (Holdridge, 1967).

Se trabajó en tres sitios de la microcuenca del río: a) parte alta - Liceo de Mata de Plátano $\left(9.955862^{\circ}\right.$ $\left.\mathrm{N}-84.018402^{\circ} \mathrm{O}\right)$, b) parte media - Parque Los Conejos $\left(9.944474^{\circ} \mathrm{N}-84.113457^{\circ} \mathrm{O}\right)$, y c) parte baja - Planta Electriona $\left(9.969228^{\circ} \mathrm{N}-84.177598^{\circ} \mathrm{O}\right)$ (figura 1$)$. 


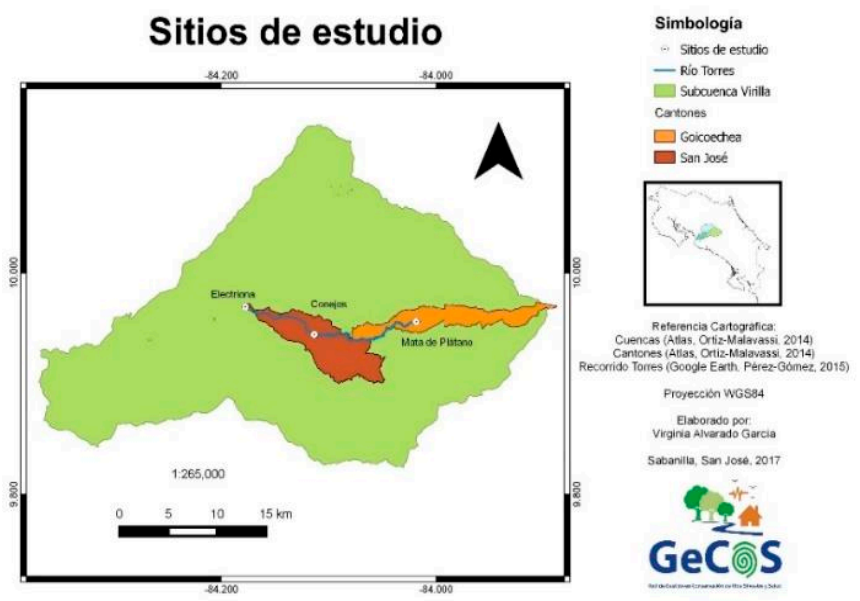

Figura 1. Sitios de estudio en el río Torres, San José, Costa Rica.

Selección de especies vegetales. Se identificaron las especies vegetales más abundantes y cercanas al río, se eligieron seis de ellas que cumplían con diversos criterios tales como: especie nativa, hábito herbáceo/arbustivo, sistema radicular profundo y fasciculado, densa cobertura, tolerancia a condiciones desfavorables, rápida propagación y reproducción asexual.

Las especies seleccionadas fueron: Lasianthaea fruticosa (L.) K.M. Becker (margarita de monte), Hamelia patens Jacq. (coralillo), Cestrum nocturnum L. (zorrillo), Dahlia imperialis Roezl ex Ortgies (catalina), Tradescantia zanonia (L.) Sw. (canutillo) y Heliconia tortuosa Griggs (platanilla).

Establecimiento de parcelas y siembra. En cada zona representativa y en laderas con pendiente entre $40^{\circ}$ y $50^{\circ}$, se instalaron ocho parcelas rectangulares de 4 × 2 m delimitadas con fibras de geotextil no tejido (GT160 de 200 $\mathrm{gr} / \mathrm{m}^{2}$ ) insertadas en el suelo unos $10 \mathrm{~cm}$ y a una altura de $30 \mathrm{~cm}$ sobre el suelo, a fin de evitar pérdidas de sedimentos. En la base de cada parcela se colocó una trampa de sedimentos con un geotextil tejido de alto módulo tipo Silt fence MacTex W2 40.

Para la siembra, se utilizó un sistema de plantación triangular o tresbolillo, en el cual las plantas se colocan a distancias iguales formando triángulos equiláteros. En el caso de los arbustos, se enterraron estacas a una profundidad mínima de $20 \mathrm{~cm}$, dejando $30 \mathrm{~cm}$ por encima del suelo; en el caso de las herbáceas se plantaron macollas o brotes originados en la base de la planta madre con medidas similares a las estacas de los arbustos.

Se establecieron ocho tratamientos (cuadro 1), en cada uno (a excepción del control) se sembraron 14 plan- tas separadas entre sí $80 \mathrm{~cm}$. La siembra se realizó en el mes de mayo del 2015, durante la transición de época seca a época lluviosa.

Cuadro 1. Tratamientos utilizados en las parcelas experimentales

\begin{tabular}{cll}
\hline Parcela & Tratamiento & Hábito \\
\hline 1 & Control & N/A \\
2 & Heliconia tortuosa Griggs & Herbácea \\
3 & Cestrum nocturnum L. & Arbusto \\
4 & Dahlia imperialis Roezl ex Ortgies & Herbácea \\
5 & Lasianthaea fruticosa (L.) K.M. Becker & Arbusto \\
6 & Tradescantia zanonia (L.) Sw. & Herbácea \\
7 & Hamelia patens Jacq. & Arbusto \\
8 & Mezcla & Todas \\
\hline
\end{tabular}

Análisis de suelo y precipitación. Se recolectaron muestras de suelo en cada sitio, a una profundidad de $20 \mathrm{~cm}$ para el análisis de textura, densidad aparente, densidad de partículas, conductividad hidráulica, porosidad, $\mathrm{pH}$, acidez, materia orgánica, capacidad de intercambio catiónico (CICE), macro y micronutrientes. El análisis se llevó a cabo en el Centro de Investigaciones Agronómicas de la Universidad de Costa Rica (CIA-UCR). Adicionalmente, se tomaron datos in situ de compactación del suelo $(\mathrm{kg} /$ $\mathrm{cm}^{2}$ ) mediante un penetrómetro de mano.

Los datos de precipitación fueron suministrados por el Instituto Meteorológico Nacional de Costa Rica (IMN) y el Instituto Costarricense de Electricidad (ICE). La intensidad de lluvia se obtuvo a partir de los datos de precipitación diaria. Las estaciones meteorológicas utilizadas fueron: CIGEFI, Aranjuez, Tobías Bolaños y Sabana Norte.

Recolección de sedimentos. A partir de la metodología de Castillo (2012) y Alvarado et al. (2013), los sedimentos retenidos en las trampas fueron recolectados en horas de la mañana y pesados in situ con una balanza de $10 \mathrm{~kg} \pm 25$ g. Posteriormente, fueron secados en un horno a $105^{\circ} \mathrm{C}$, y finalmente pesados en una balanza analítica. La tasa de erosión se cuantificó a partir de la producción de sedimentos durante seis meses (julio a diciembre del 2015).

Sobrevivencia. El porcentaje de sobrevivencia se calculó por presencia/ausencia de estacas o macollas en cada parcela. En el caso de que las plántulas no presentaran hojas, se hizo un leve raspado en el tallo para constatar que estuvieran vivas (color verde) o muertas (color café). Esto se hizo durante siete meses (junio a diciembre del 2015). 
Análisis de datos. Para determinar diferencias entre la retención de sedimentos en tratamientos vegetales y el control, se corrió un análisis de varianza no paramétrica (Kruskall Wallis); asimismo, para evaluar la producción de sedimentos y el porcentaje de sobrevivencia en los tratamientos vegetales se utilizó un modelo lineal generalizado con distribución de Poisson. Ambos en el programa R commander. Además, se realizó un análisis de similitud en el programa Past $2.17 \mathrm{c}$ para evaluar la semejanza entre los tratamientos aplicados.

\section{RESULTADOS Y DISCUSIÓN}

En términos generales, las propiedades físicas y químicas del suelo se encontraron dentro de los niveles óptimos, exceptuando la conductividad hidráulica, que fue alta en
El intercambio catiónico es un indicador del potencial del suelo para retener e intercambiar nutrientes y está relacionado con las arcillas y la materia orgánica, las cuales tienen carga negativa. En los tres sitios, los valores de la CICE fueron óptimos, lo cual es fundamental para el establecimiento y desarrollo de coberturas vegetales (Ruales y Ruíz 2006), y por ende, una mayor estabilidad estructural del suelo (Suárez 2001).

Con respecto a la textura, hubo predominancia de partículas gruesas; sin embargo, la combinación con partículas más finas contribuyó a una mayor facilidad de disgregación y lavado del material, pero en distancias cortas. Según Echeverría et al. (2006), los terrenos con textura fina son más resistentes a la desagregación, pero son más fáciles de transportar; caso contrario ocurre con las textu-

Cuadro 2. Propiedades fisicoquímicas del suelo en las parcelas experimentales

\begin{tabular}{|c|c|c|c|c|c|c|c|c|c|}
\hline \multicolumn{10}{|c|}{ Análisis físico } \\
\hline Sitios de estudio & $\begin{array}{c}\text { Densidad } \\
\text { aparente }\end{array}$ & $\begin{array}{l}\text { Densidad } \\
\text { partículas }\end{array}$ & $\begin{array}{l}\text { Conduct. } \\
\text { hidráulica }\end{array}$ & Porosidad & \multicolumn{4}{|c|}{ Textura $(\%)$} & Compactación \\
\hline & $\mathrm{g} \mathrm{cm}^{-3}$ & $\mathrm{~g} \mathrm{~cm}^{-3}$ & $\mathrm{~cm} \mathrm{~h}^{-1}$ & $\%$ & Arena & Limo & Arcilla & Clase & $\mathrm{kg} / \mathrm{cm}^{2}$ \\
\hline Mata de plátano & 1.01 & 2.34 & 768 & 56.84 & 70 & 10 & 20 & $\begin{array}{l}\text { Franco } \\
\text { arenoso }\end{array}$ & 0.75 \\
\hline Conejos & 1.02 & 2.43 & 152 & 58.02 & 72 & 3 & 25 & $\begin{array}{c}\text { Franco arcillo } \\
\text { arenoso }\end{array}$ & 2.00 \\
\hline Electriona & 1.08 & 2.40 & 1330 & 55.00 & 60 & 10 & 30 & $\begin{array}{c}\text { Franco arcillo } \\
\text { arenoso }\end{array}$ & 2.00 \\
\hline
\end{tabular}

Análisis químicos

\begin{tabular}{|c|c|c|c|c|c|c|c|c|c|c|c|c|}
\hline \multirow[b]{2}{*}{ Sitios de estudio } & $\mathrm{pH}$ & Acidez & $\mathrm{Ca}$ & $\mathrm{Mg}$ & $\mathrm{K}$ & CICE & $\mathrm{P}$ & $\mathrm{Zn}$ & $\mathrm{Cu}$ & $\mathrm{Fe}$ & $\mathrm{Mn}$ & $\mathrm{MO}$ \\
\hline & $\mathrm{H}_{2} \mathrm{O}$ & \multicolumn{5}{|c|}{$\operatorname{cmol}(+) / \mathrm{L}$} & \multicolumn{5}{|c|}{$\mathrm{mg} / \mathrm{L}$} & $\%$ \\
\hline Mata de plátano & 6.2 & 0.18 & 5.02 & 2.79 & 1.26 & 9.25 & 5 & 4.1 & 25 & 216 & 13 & 4.97 \\
\hline Conejos & 6.9 & 0.11 & 10.35 & 2.83 & 1.86 & 15.15 & 31 & 5.2 & 32 & 111 & 10 & 1.61 \\
\hline Electriona & 6.3 & 0.12 & 9.96 & 3.53 & 0.68 & 14.29 & 3 & 8.4 & 15 & 144 & 15 & 5.10 \\
\hline
\end{tabular}

$\mathrm{CICE}=$ Capacidad de intercambio catiónico efectivo, $\mathrm{MO}=$ Materia orgánica

Fuente: Centro de Investigaciones Agronómicas, UCR, 2015.

Electriona y baja en Conejos. La compactación fue doblemente mayor en Conejos y Electriona (cuadro 2).

El sector de Mata de Plátano presentó una profundidad efectiva alta $(>120 \mathrm{~cm})$, especies leñosas y herbáceas con abundantes raíces en niveles inferiores. En el sector de Conejos, la profundidad efectiva se vio limitada por una mala estructura debido a la cantidad de escombros; mientras que en Electriona, las raíces se encentraron en niveles superiores debido a una profundidad efectiva media $(<60 \mathrm{~cm})$. Esto puede explicar la alta compactación en las zonas más bajas del río. ras gruesas. Específicamente en suelos francos, como en este caso, son fácilmente erodables, pues son más susceptibles a ser disgregados y transportados.

De los factores que más influyen en la erosión del suelo, el más determinante es la precipitación (Wei et al. 2009); la intensidad, duración y frecuencia de ésta, definen el poder erosivo sobre un terreno (Núñez 2001). Los meses más lluviosos durante el estudio fueron octubre y noviembre, donde Mata de Plátano registró la precipitación más alta de los tres sitios. Por su parte, diciembre fue el mes más seco (cuadro 3). 
Cuadro 3. Precipitación mensual durante el período de estudio

\begin{tabular}{lrcr}
\hline \multicolumn{1}{c}{ Mes } & Mata de Plátano & Conejos & Electriona \\
\cline { 2 - 4 } & \multicolumn{3}{c}{ Precipitación $(\mathrm{mm})$} \\
Junio & 168.50 & 165.20 & 153.10 \\
Julio & 181.30 & 200.00 & 136.40 \\
Agosto & 61.80 & 21.10 & 98.50 \\
Setiembre & 125.50 & 135.40 & 103.20 \\
Octubre & 430.20 & 256.00 & 290.30 \\
Noviembre & 215.40 & 182.60 & 244.80 \\
Diciembre & 50.30 & 51.80 & 35.00 \\
\hline Total & 1233.00 & 1012.10 & 1061.30 \\
\hline
\end{tabular}

Fuente: IMN e ICE, 2015.

Una de las características más importantes de la lluvia es su intensidad (Muñoz y Ritter 2005) y se estima que todo evento pluvial con intensidad menor de $25 \mathrm{~mm}$ $\mathrm{h}^{-1}$ no se considera erosivo (Núñez 2001). Según De Alba et al. (2011), intensidades bajas a medias ocasionan tasas de erosión de 7.3 ton $h^{-1} a^{-1} o^{-1} y$ se pierde una lámina de suelo de $0.5 \mathrm{~mm}$.

Los valores de intensidad fueron similares en los tres sitios; sin embargo, Mata de Plátano reportó los valores de intensidad más altos. Se registraron intensidades máximas por encima de $36 \mathrm{~mm} \mathrm{~h}^{-1}$ únicamente en octubre; el resto de meses osciló entre los 6 y $35 \mathrm{~mm} \mathrm{~h}^{-1}$. A pesar de que diciembre fue el mes con menor precipitación, reportó intensidades similares a setiembre (cuadro 4).

Cuadro 4. Intensidad máxima durante el período de estudio

\begin{tabular}{lccc}
\hline \multicolumn{1}{c}{ Mes } & Mata de Plátano & Conejos & Electriona \\
\hline \multirow{2}{*}{ Julio } & \multicolumn{2}{c}{ Intensidad máxima $\left(\mathrm{mm} \mathrm{h}^{-1}\right)$} \\
\cline { 2 - 4 } Agosto & 19.00 & 27.4 & 22.0 \\
Setiembre & 30.30 & 6.6 & 34.0 \\
Octubre & 9.30 & 21.8 & 17.1 \\
Noviembre & 58.20 & 36.8 & 41.1 \\
Diciembre & 33.30 & 19.8 & 35.9 \\
\hline
\end{tabular}

Fuente: INM e ICE, 2015.

De Alba et al., (1998), determinaron que los episodios extremos de lluvia ocasionan más de un $80 \%$ de las tasas de erosión medias anuales en el mediterráneo. A pesar de ello, en el trópico los eventos extremos de intensidad de lluvia (>120 mm h-1) se consideran altamente erosivos (Núñez, 2001).

Según De Alba et al., (2011), un episodio extremo de intensidad de lluvia puede ocasionar una tasa de erosión de 39 ton ha $^{-1} \mathrm{año}^{-1}$ A pesar de que los sitios evidenciaron una erosión severa a muy severa, no se reportó intensidades máximas tan altas, por lo que la producción de sedimentos pudo deberse a otros factores como la pendiente y el uso del suelo.

La producción de sedimentos fue variable en los tres sitios, siendo Conejos el lugar con mayor sedimentación. En la parte alta y baja se registraron tasas de erosión por debajo de las 65 ton/ha, en tanto que, en la parte media, este valor fue duplicado (cuadro 5).

Cuadro 5. Tasa de erosión promedio durante el período de estudio

\begin{tabular}{llccc}
\hline & & \multicolumn{1}{c}{$\begin{array}{c}\text { Mata de } \\
\text { Plátano }\end{array}$} & Conejos & Electriona \\
\cline { 3 - 5 } & Tratamientos & \multicolumn{2}{c}{$\begin{array}{c}\text { Tasa de erosión } \\
\left.\text { (ton } \text { ha }^{-1} \text { año }^{-1}\right)\end{array}$} \\
\hline 1 & Control & 11.79 & 11.51 & 7.75 \\
2 & H. tortuosa & 8.09 & 19.67 & 10.87 \\
3 & C. nocturnum & 4.36 & 21.76 & 9.92 \\
4 & D. imperialis & 7.04 & 8.16 & 11.29 \\
5 & L. fruticosa & 5.32 & 36.65 & 5.25 \\
6 & T. zanonia & 4.24 & 9.25 & 0.56 \\
7 & H. patens & 2.86 & 12.33 & 3.28 \\
8 & Mezcla & 4.27 & 7.72 & 14.96 \\
\hline & Total & $47.97 *$ & $127.05^{* *}$ & $63.88^{* *}$ \\
\hline
\end{tabular}

* Erosión severa, ** Erosión muy severa

En la parte alta y baja, las parcelas sembradas con $H$. patens y T. zanonia, registraron menos tasa de erosión. Para la parte media, $D$. imperialis y $T$. zanonia, fueron las más eficientes en comparación a las otras cuatro especies.

Mata de Plátano conserva un bosque ribereño mayor que Conejos, por lo que se evidenció una clara diferencia entre las tasas de erosión del control y el resto. Según Sepúlveda et al. (2009), los bordes de ríos con presencia de árboles son menos susceptibles a la erosión que zonas ribereñas con vegetación dispersa. Por otro lado, Electriona también reportó tasas de erosión mucho menores que Conejos; sin embargo, la cobertura a su alrededor está predominada por pastos y según Zaimes et al. (2005), las pérdidas por erosión fluvial son significativamente mayores, bajo esas condiciones.

El análisis de varianza evidenció que no existen diferencias significativas entre los tratamientos vegetales y el control $(\mathrm{KW}=23, \mathrm{gl}=23, \mathrm{p}>0.05)$; sin embargo, sí las hay entre los tratamientos vegetales (AIC $=267.3, \mathrm{gl}$ $=20, \mathrm{p}<0.05$ ). Esto pudo deberse a que las condiciones del sitio afectaron el establecimiento de ciertas especies y muchas parcelas actuaron como otro control. 

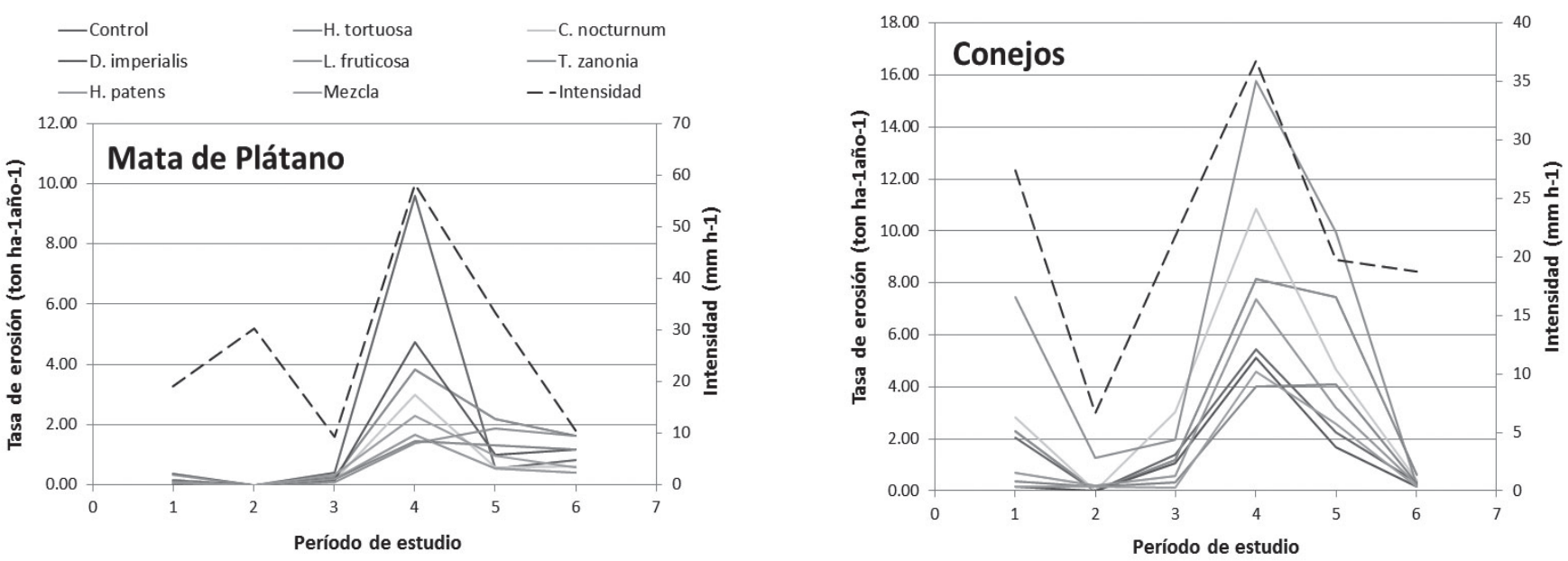

A pesar de ello, para las condiciones del estudio, se observó un incremento en la tasa de erosión al aumentar la intensidad, lo cual evidenció una relación entre ambos (figura 2).

Al igual que la precipitación, la presencia o ausencia de cobertura vegetal influye en los procesos erosivos. La vegetación aumenta la estabilidad de los agregados del suelo, lo protege del impacto de las gotas de lluvia, incrementa la capacidad de infiltración y frena la escorrentía (Alvarado, 2016).

Según Morgan (2005), la efectividad de la vegetación para disminuir la producción de sedimentos depende directamente de la altura, continuidad y densidad de las especies vegetales; sin embargo, también de las características del lugar y de las especies a utilizar (Alvarado et al., 2013).

En Mata de Plátano fue: $7>6>8>3>5>4>2$. H. patens (arbusto), T. zanonia (herbácea), C. nocturnum (arbusto) y $L$. fruticosa (arbusto) comparten mayor similitud entre sí, por lo que, en este sitio, los arbustos tuvieron mayor éxito (figura 3 ).

El orden de efectividad vegetal en el sector de Conejos fue: $8>4>6>7>2>3>5$. En este sitio las herbáceas tuvieron mayor éxito (figura 4 ); $D$. imperialis (herbácea) y $T$. zanonia (herbácea) comparten similitudes con $H$. patens (arbusto).
El orden de efectividad vegetal en el sector de Electriona fue: $6>7>5>3$ $>2>4>8$. Hay dos agrupaciones bien marcadas, una está dominada por herbáceas y la otra por arbustos. A pesar que la especie más efectiva fue $T$. zanonia (herbácea), ésta comparte similitudes con $H$. patens y $L$. fruticosa, por lo que en este sitio los arbustos tuvieron mayor éxito (figura 5).

En general, en la parte alta y baja, predominaron los arbustos; mientras

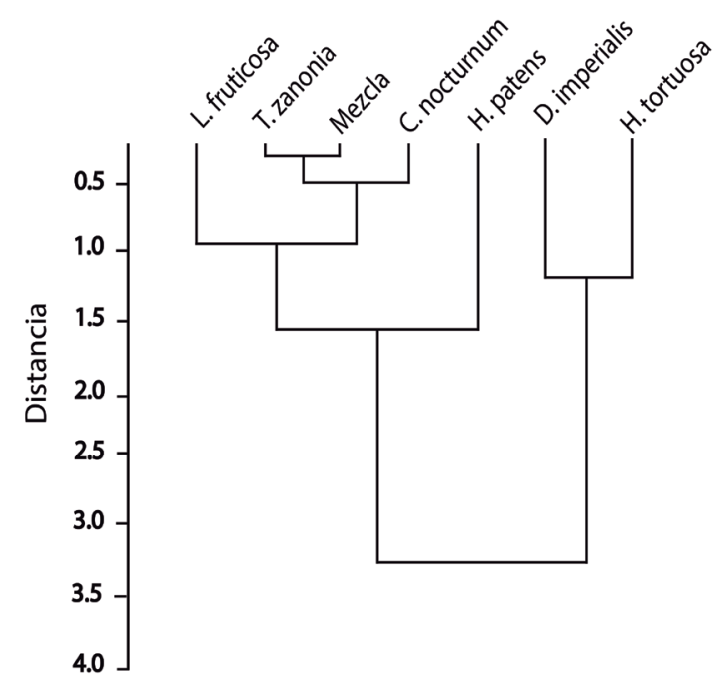

Figura 3. Análisis similitud entre tratamientos vegetales, Mata de Plátano. 


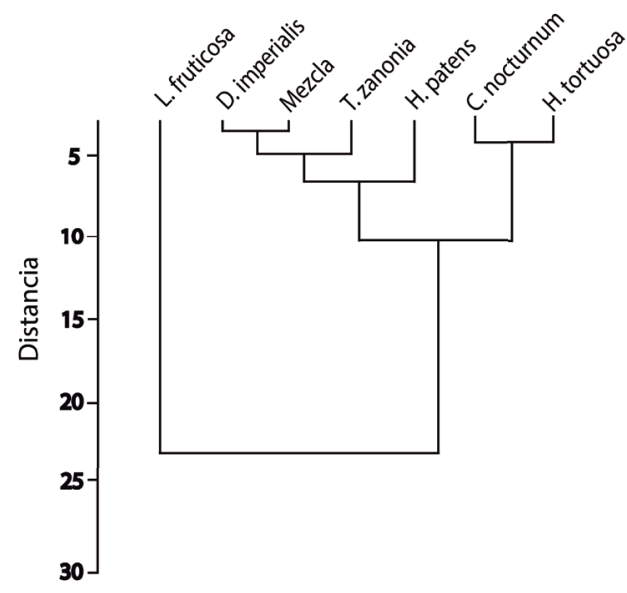

Figura 4. Análisis similitud entre tratamientos vegetales, Conejos.

que, en la media, las herbáceas. En el caso de Electriona, los pastos no le ofrecen una estabilidad estructural, ya que la vegetación herbácea posee una gran densidad de raíces finas que se distribuyen en la parte superficial (primeros $30 \mathrm{~cm}$ del suelo) y en horizontes más profundos puede ocurrir un fuerte estrés hidráulico (Sepúlveda et al., 2009). Por lo que, en cuanto a la efectividad vegetal, los arbustos fueron más exitosos, debido a que sus raíces sí logran

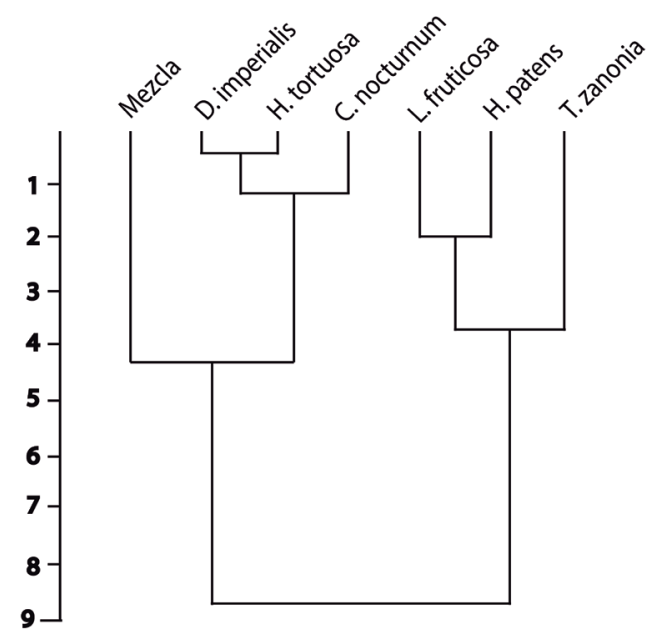

Figura 5. Análisis similitud entre tratamientos vegetales, Electriona.

distribuirse más ampliamente en el perfil del suelo y le confieren mayor resistencia hidráulica al terreno, lo cual se traduce en una menor erosión.

Por el contrario, Conejos es una zona ribereña perturbada, lo cual la hace más vulnerable a la erosión debido al socavamiento de material. Bajo estas condiciones, la pérdida de suelo puede ser muy severa sobretodo en climas tropicales con fuertes lluvias (Núñez, 2001; Sepúlveda et al., 2009). Es por ello que la efectividad vegetal estuvo liderada por herbáceas, las cuales cumplen un rol de control, debido la densidad y disposición de su sistema radicular (Suárez, 2001).

El hecho de que sea mejor utilizar herbáceas en Conejos no puede quedar ahí; sino que una vez establecida la vegetación del terreno, se debe introducir una variedad de especies leñosas con tasa diferente de crecimiento y tamaño (Rondón y Vidal, 2005), para formar estratos y mejorar la funcionalidad del ecosistema.

En cuanto a la sobrevivencia, la parte alta y media mostraron un porcentaje mayor, respecto a la baja (cuadro 6). En los tres sitios, T. zanonia y H. tortuosa registraron una sobrevivencia mayor al $70 \%$.

Cuadro 6. Porcentaje de sobrevivencia durante el período de estudio

\begin{tabular}{lccc}
\hline \multicolumn{1}{c}{ Tratamiento } & Mata de Plátano & Conejos & Electriona \\
\hline & \multicolumn{2}{c}{ Porcentaje de sobrevivencia (\%) } \\
\cline { 2 - 4 } H. tortuosa & 73.46 & 93.86 & 77.54 \\
C. nocturnum & 73.45 & 65.27 & 44.89 \\
D. imperialis & 92.86 & 54.06 & 53.04 \\
L. fruticosa & 46.92 & 34.69 & 21.40 \\
T. zanonia & 70.40 & 87.74 & 77.54 \\
H. patens & 63.24 & 45.90 & 17.34 \\
Mezcla & 49.98 & 70.40 & 60.18 \\
\hline Promedio & 67.19 & 64.56 & 50.28 \\
\hline
\end{tabular}

En la parte alta, $D$. imperialis registró el mayor porcentaje de sobrevivencia $(>90 \%)$ y fue altamente significativa con respecto al resto $(\mathrm{AIC}=165.13, \mathrm{gl}=20, \mathrm{p}<$ 0.05 ). La sobrevivencia en esta zona se consideró exitosa debido a las condiciones edafológicas y climáticas del lugar.

En la parte media se registró diferencias en $H$. tortuosa $(\mathrm{AIC}=165.13, \mathrm{gl}=20, \mathrm{p}<0.05), T$. zanonia $(\mathrm{AIC}=165.13, \mathrm{gl}=20, \mathrm{p}<0.05)$ y $C$. nocturnum $(\mathrm{AIC}=$ 165.13, $\mathrm{gl}=20, \mathrm{p}<0.05)$. La sobrevivencia en esta zona se consideró aceptable debido a que la estructura del suelo no es la adecuada, puesto que está inmersa entre escombros de construcción y desechos sólidos.

En la parte baja, las diferencias se observaron en H. patens $(\mathrm{AIC}=165.13, \mathrm{gl}=20, \mathrm{p}<0.05)$, L. fruticosa $(\mathrm{AIC}=165.13, \mathrm{gl}=20, \mathrm{p}<0.05)$ y $C$. nocturnum $(\mathrm{AIC}=$ $165.13, \mathrm{gl}=20, \mathrm{p}<0.05)$, siendo éstas las que reportaron menor sobrevivencia $(<45 \%)$. La sobrevivencia en esta zona se consideró aceptable pero más baja que el resto, debido a las condiciones edafológicas y climáticas del lugar. 
El hecho de que muchas especies no hayan sido tan exitosas se debió principalmente a la heterogeneidad de las precipitaciones. A pesar de que se sembró a inicios de la época lluviosa, el clima fue atípico. Esto ocasionó que el material vegetativo entrara en un proceso de pudrición, principalmente en $L$. fruticosa, lo que aumentó su tasa de erosión.

En general, la sobrevivencia promedio en los tres sitios estuvo por encima del $50 \%$, lo cual evidencia que las especies superaron el estrés post siembra y lograron adaptarse exitosamente a las condiciones del entorno (Hernández et al., 2010).

Para asegurar el éxito de las especies nativas en cuanto a control de erosión y restauración ecológica, es necesario conocer sus atributos fisiológicos, morfológicos y botánicos que determinan su respuesta; al igual que la intensidad de la perturbación y las condiciones ambientales del sitio.

El uso de una cubierta vegetal con especies nativas de herbáceas y arbustos, como estrategia de restauración ecológica y foresta urbana, constituye una herramienta integral para mejorar las condiciones mecánicas, hidráulicas y ambientales del sitio de estudio, y actúa como barrera viva contra la erosión.

No obstante, debido al carácter descriptivo y exploratorio de estos resultados, queda abierta la necesidad de nuevos estudios orientados a validar el potencial de estas y otras especies como factor de control de la erosión y restauración ecológica en otras zonas del país, bajo condiciones climáticas y topográficas diferentes.

\section{CONCLUSIONES}

De las herbáceas, la que presentó mayor sobrevivencia y menor tasa de erosión fue T. zanonia; de los arbustos, fue $H$. patens. Esto puede ser de gran utilidad para poner a prueba una asociación multiestratificada de especies con múltiples usos. Dado que el sitio de estudio es un río urbano y potencialmente, un corredor fluvial verde, estas especies serían fundamentales desde el punto de vista estructural, ambiental y económico.

En general, en la parte alta y baja, predominaron los arbustos; mientras que, en la media, las herbáceas. El uso de especies nativas como estrategia de restauración ecológica y foresta urbana permite mejorar las condiciones mecánicas, hidráulicas y ambientales del sitio de estudio.

\section{AGRADECIMIENTOS}

Extendemos nuestro agradecimiento a la Universidad Estatal a Distancia por el aval para llevar a cabo este proyecto; así como a la Planta Hidroeléctrica Electriona, al Liceo de Mata de Plátano, al Instituto Costarricense de Electricidad, al Instituto Meteorológico Nacional de Costa Rica y al Centro de Investigaciones Agronómicas de la Universidad de Costa Rica por su colaboración.

\section{REFERENCIAS BIBLIOGRÁFICAS}

Alvarado, V. 2016. La vegetación como factor de control de la erosión. Repertorio Científico 19(1):13-17.

Alvarado, V., Bermúdez, T., Romero, M., Piedra, L. 2013. Evaluación de la revegetación para el control de la erosión laminar en taludes de la Microcuenca del Río Pirro, Costa Rica. Tesis de Licenciatura, Universidad Nacional, Costa Rica.

Bochet, E., García, P. 2004. Factors controlling vegetation establishment and water erosion on Motorway Slopes in Valencia, Spain. Restoration Ecology 12(2):166-174.

Castillo, M. 2012. Determinación y cuantificación de la tasa de erosión en un ciclo del cultivo de papa en la zona de Pacayas de Alvarado, Cartago. Tesis de Licenciatura, Universidad de Costa Rica, Costa Rica.

Conejero, A., Sallent, C. 2011. Estudio del ecosistema urbano de San José. Protocolo de monitoreo de aves y naturalización del Parque La Sabana. Universidad Nacional., Heredia, Costa Rica. Disponible en: http://ddd.uab.cat/pub/trerecpro/2011/hdl_2072_179283/ PFC_EcosistemaUrbaSanJose.pdf

De Alba, S., Benito, G., Pérez, A. 1998. Erosión de suelo en episodios de lluvia de elevada intensidad versus episodios de moderada y baja intensidad y elevada frecuencia en ambientes semiáridos. En: Gómez- Ortiz, A., Salvador-Franch, F. (eds.). Investigaciones Recientes de la Geomorfología Española, pp. 483-492. Barcelona, España.

De Alba, S., Alcázar, M., Cermeño, F., Barbero, F. 2011. Erosión y manejo de suelo. Importancia de Laboreo ante los procesos erosivos naturales y antrópicos. Agricultura Ecológica en Secano 1(7):13-38.

Echeverría, N., Vallejos, A., Silenzi, J. 2006. Erodabilidad de suelos del sur de la Región Semiárida Argentina. Ci Suelo 24(1):49-57.

Feoli, S. 2013. Corredor Biológico Interurbano del Río Torres y corredores biológicos en general. Ambientico 232-233:51-55.

Hernández-Sandoval, L., González-Hernández, M.E., Malda-Barrera, G., Suzán, H., Bustos-Contreras, D.E., Terrones-Rincón, R. 2010. Establecimiento y crecimiento en las primeras etapas de diez especies arbustivas nativas, en sitios deforestados de la microcuenca de Santa Rosa Jáuregui, Querétaro, México. Ciencia@uaq 3(2):27-41. 
Holdridge, L.R. 1967. Life zone ecology. Tropical Science Center, San José, Costa Rica. Disponible en: http://reddcr.go.cr/sites/default/ files/centro-de-documentacion/holdridge_1966_-life_zone_ecology.pdf

Laporte, G., Porras, G. 2002. Uso de la vegetación para la estabilización de taludes. En VIII Seminario Nacional de Geotecnia, III Encuentro Centroamericano de Geotecnistas. pp.18. San José, Costa Rica.

Mendoza, A. 2012. Sábado de reforestación en La Sabana. Disponible en: http://www.crhoy.com/sabado-de-reforestacion/

Morgan, R. 2005. Soil erosion and conservation. Blackwell Publishing Ltd., Oxford, UK.

Muñoz, R., Ritter, A. 2005. Hidrología agroforestal. Mundi-Prensa, Madrid, España.

Núñez, J. 2001. Manejo y conservación de suelos. EUNED, San José, Costa Rica.

Porras, G. 2000. Uso de la vegetación para la estabilización de taludes. Tesis de Licenciatura, Universidad de Costa Rica, Costa Rica.

Romero, M., Piedra, L., Villalobos, R., Marín, R., Núñez, F. 2011. Evaluación ecológica rápida de un ecosistema urbano: El caso de la microcuenca del río Pirro Heredia, Costa Rica. Revista Geográfica de América Central 47:41-69.

Rondón, J., Vidal, R. 2005. Establecimiento de la cubierta vegetal en áreas degradadas (Principios y métodos). Revista Forestal Latinoamericana (38):63-82.

Ruales, P., Ruíz, J.F. 2006. Necesidades nutricionales de la alcachofa (Cynara scolymus L.) en dos tipos de suelo en dos localidades. Tesis de Ingeniería Agropecuaria, Sangolquí, Ecuador.

Sancho, F., Cervantes, C. 1997. El uso de plantas de cobertura en sistemas de producción de cultivos perennes y anuales en Costa Rica. Agronomía Costarricense 21(1):111-120.

Sepúlveda-Lozada, A., Geissen, V., Ochoa-Gaona, S., Jarquín-Sánchez, A., Hernández de la Cruz, S., Capetillo, E., Zamora-Cornelio, L.F. 2009. Influencia de tres tipos de vegetación ribereña en el control de la erosión fluvial en Pantanos de Centla, México. Rev. Biol. Trop. 57(4):1153-1163.

Suárez, J. 2001. Control de erosión en zonas tropicales. Universidad Industrial de Santander, Bucaramanga, Colombia.

Villar, R., Ruiz-Robleto, J., Quero, J.L., Poorter, H., Valladares, F., Marañón, T. 2004. Tasas de crecimiento en especies leñosas: aspectos funcionales e implicaciones ecológicas. En: Valladares, F. (eds.), Ecología del bosque mediterráneo en un mundo cambiante, pp. 191-227. EGRAF, S. A., Madrid, España.

Wei, L., Chen, L. Fu, B. 2009. Effects of rainfall change on water erosion processes in terrestrial ecosystems: a review. Progress in Physical Geography 33(3):307-318.

Zaimes, G.N., Schultz, R.C., Isenhart, T.M., Mickelson, S.K., Kovar, J.L., Russell, J.R., Powers, W.P. 2005. Stream bank erosion under different riparian land-use practices in northeast Iowa. En: Brooks, K.N., Ffolliott, P.F. (eds.), Moving agroforestry into the mainstream. The 9th North American Agroforestry Conference Proceedings. Rochester, Minesota, EEUU.

Zúñiga, M.A. 2016. Caracterización de la vegetación nativa para la restauración ecológica y foresta urbana de la microcuenca del río Torres, Costa Rica. Tesis de Licenciatura, Universidad Estatal a Distancia, Costa Rica.

Zwart, M., Rojo, J., de la Cruz, R., Yeomans, J. 2005. Coberturas y la salud del suelo. Tierra tropical 1(1):9-20. 